\title{
Dupla variação anatômica vascular em um único indivíduo: estudo morfométrico
}

\author{
Dual vascular anatomical variation in a single individual: morphometric study
}

Cristiane Regina Ruiz ${ }^{1}$, Sergio Ricardo Rios Nascimento ${ }^{1}$, Alex Kors Vidsiunas ${ }^{1}$, Lilian Andrades², Cristiano Cirqueira de Souza ${ }^{3}$

${ }^{1}$ Curso de Especialização em Anatomia Macroscópica e por Imagens, Centro Universitário São Camilo (CUSC) - São Paulo (SP), Brasil.

2Laboratório de Anatomia Humana, CUSC - São Paulo (SP), Brasil.

${ }^{3}$ Curso de Tecnologia em Radiologia, CUSC - São Paulo (SP), Brasil.

DOI: http://dx.doi.org/10.7322/abcshs.v42i3.919

\section{RESUMO}

Introdução: Variações anatômicas são pequenas diferenças morfológicas congênitas que aparecem nos diferentes sistemas orgânicos, as quais não acarretam prejuízo ou distúrbio funcional para o indivíduo. No que diz respeito aos vasos sanguíneos, alterações no desenvolvimento embriológico podem gerar duplicidade de vasos, agenesia ou ocasionar o surgimento de artérias e o desembocar de veias fora da descrição anatômica padrão. Relato de caso: Foi observada dupla variação anatômica vascular em um indivíduo durante uma dissecação de rotina no Laboratório de Anatomia do Centro Universitário São Camilo. A artéria renal principal tinha origem na parte abdominal da artéria aorta seguindo até sua entrada no hilo renal, porém, em vez de um trajeto retilíneo a partir da aorta, a mesma possuía um trajeto descendente e bem angulado. A partir da artéria renal principal surgia uma artéria polar aberrante que entrava no polo inferior do rim direito. Em um nível mais inferior, na altura da bifurcação da aorta, originava-se outra artéria polar aberrante que entrava no hilo renal, seguindo um trajeto retilíneo até o polo inferior do rim direito. A artéria hepática comum originava-se no tronco celíaco seguindo até sua entrada na porta do fígado. A artéria mesentérica superior possuía origem no tronco celíaco. A artéria hepática direita originava-se na artéria mesentérica superior. Conclusão: $O$ estudo das variações anatômicas constitui-se tarefa árdua em virtude das inúmeras expressões diferentes que ocorrem no corpo humano, porém, o conhecimento dessas variações é de extrema valia para a clínica e o planejamento cirúrgico, garantindo a precisão e evitando complicações pós-cirúrgicas ou diagnósticos errôneos.

Palavras-chave: Variação anatômica; morfologia; vasos sanguíneos; abdome.

\begin{abstract}
Introduction: Anatomical variations are small congenital morphological differences that appear in different organ systems, which do not result in damage or functional disturbance for the individual. When it comes to blood vessels, alterations in embryological development may lead to the duplicity and the agenesis of vessels, or cause the emergence of arteries and the disembogue of vessels not according to the standard anatomical description. Case report: Dual anatomical variation in an individual was observed during a routine dissection in the Laboratory of Anatomy of the Centro Universitário São Camilo. The main renal artery originated in the abdominal part of the aorta artery, following its path until its entry in the renal hilum. However, instead of a rectilinear path from the aorta, it had a descending and well-angulated path. From the main renal artery, an aberrant polar artery emerged, that entered the inferior pole of the right kidney. At a lower level, on the same level as the bifurcation of the aorta, another aberrant polar artery emerged, which entered the renal hilum following a straight path to the inferior pole of the right kidney. The common hepatic artery was originated in the celiac trunk, following its path until its entrance in the porta hepatis. The superior mesenteric artery was originated in the celiac trunk. The right hepatic artery was originated in the superior mesenteric artery. Conclusion: The study of anatomical variations is an arduous task due to the innumerable different expressions that occur in the human body. However, the knowledge of these variations is extremely valuable for medicine and surgical planning, assuring accuracy and avoiding postoperative complications or erroneous diagnoses.
\end{abstract}

Keywords: anatomical variation; morphology; blood vessels; abdomen.

Recebido em: 06/01/2017

Revisado em: $17 / 09 / 2017$

Aprovado em: 16/10/2017

Autor para correspondência: Sergio Ricardo Rios Nascimento - Centro Universitário São Camilo - Avenida Nazaré, 1501 - Ipiranga - CEP: $04263-200$ São Paulo (SP), Brasil - E-mail: srrnascimento@ gmail.com

Conflito de interesse: nada a declarar. 


\section{INTRODUÇÃO}

Variações anatômicas são pequenas diferenças morfológicas congênitas que aparecem nos diferentes sistemas orgânicos, as quais não acarretam prejuízo ou distúrbio funcional para o indivíduo ${ }^{1}$. No que diz respeito aos vasos sanguíneos, alterações no desenvolvimento embriológico podem gerar duplicidade de vasos, agenesia ou ocasionar o surgimento de artérias e o desembocar de veias fora da descrição anatômica padrão. Malformações no desenvolvimento dos arcos aórticos podem causar uma diferença na disposição habitual do trajeto dos vasos sanguíneos ${ }^{1,2}$.

As artérias renais, oriundas da parte abdominal da aorta, possuem variações em número, origem, ramificação e trajeto que não são raras $^{2}$. De acordo com Ozkan et al. ${ }^{3}$, a formação padrão clássica de uma artéria e uma veia de cada lado do indivíduo aparece em menos de $25 \%$ dos casos. Apesar da frequência de variações ser alta, há uma grande quantidade de diferenças anatômicas em cada uma, tornando sua documentação de extrema importância para a clínica cirúrgica, para o diagnóstico por imagem, além da área acadêmica durante a formação de novos profissionais ${ }^{4-8}$.

Há artérias acessórias, que seguem para o rim pelo hilo, e artérias aberrantes, que suprem o rim sem entrar pelo hilo ${ }^{8}$. Essas artérias podem se direcionar para o polo superior ou para o polo inferior, sendo nesses casos denominadas artérias polares ${ }^{3}$.

O conhecimento da existência dessas artérias é importante para evitar lesões durante cirurgias renais e a presença das mesmas deve ser considerada em casos de doação de órgãos e transplantes renais, visto que rins com múltiplas artérias podem resultar em morbidade pós-transplante ${ }^{8,9}$.

Variações anatômicas nas artérias hepáticas e no tronco celíaco são de considerável importância em transplantes hepáticos, cirurgias laparoscópicas, intervenções radiológicas abdominais e lesões perfurantes no abdome ${ }^{10-13}$.

É sabido que o padrão de suprimento arterial para o fígado é variável, sendo que modificações na organização padrão pela qual o fígado recebe seu fluxo total de sangue a partir de ramos do tronco celíaco ocorrem em 25 a 75\% dos casos ${ }^{10}$.

O tronco celíaco, que normalmente dá origem às artérias gástrica esquerda, hepática comum e esplênica, também pode em alguns casos dar origem às artérias frênicas inferiores e à artéria mesentérica superior ${ }^{1}$. Variações nos ramos do tronco celíaco são descritas na literatura, bem como a probabilidade de que o percentual de cadáveres com variações seja de 22,5 a 57,5\% ${ }^{2}$.

$\mathrm{Na}$ literatura encontramos citações diversas sobre artérias hepáticas acessórias, quando a mesma leva sangue para um lobo já irrigado por sua artéria normal, e artéria hepática substituta, que é um vaso que não tem uma origem ortodoxa e irriga sozinha um lobo. Quando presente, a artéria hepática acessória, ou ramo direito acessório da artéria hepática, tem origem na artéria mesentérica superior ${ }^{1}$.

Vários autores descreveram variações na origem e no trajeto da artéria hepática, inclusive criando classificações específicas ${ }^{10,14,15}$. Com o avanço dos métodos de imagem pudemos aumentar a quantidade de indivíduos estudados sem a necessidade da dissecação, fato que auxiliou exponencialmente os estudos quantitativos que determinam um padrão das variações em determinadas populações.

Em uma dissecação de rotina no Laboratório de Anatomia do Centro Universitário São Camilo, com o intuito de retirada de alguns órgãos internos de um cadáver masculino, foram observadas múltiplas variações em vasos sanguíneos da cavidade abdominal e pélvica. Nosso objetivo é descrever morfometricamente as variações anatômicas encontradas nas artérias renais, hepática e mesentérica superior e demonstrá-las por meio de documentação fotográfica e ilustrações esquemáticas.

\section{RELATO DE CASO}

A primeira variação encontrada foi na quantidade das artérias renais, bem como na origem das mesmas, sendo que o rim direito demonstrava diferença visível em relação ao rim esquerdo em sua forma, tamanho e localização.

O rim direito possuía largura de $36,35 \mathrm{~mm}$ em sua face anterior e largura de 52,16 mm em sua face posterior, com comprimento de 141,22 $\mathrm{mm}$. O polo inferior do rim direito estava em contato com a margem superior da crista ilíaca direita. A artéria renal principal tinha origem na parte abdominal da artéria aorta e seguia um curso de $81,66 \mathrm{~mm}$ até sua entrada no hilo renal, com um calibre de $6,54 \mathrm{~mm}$, porém, em vez de um trajeto retilíneo a partir da aorta, a mesma possuía um trajeto descendente e bem angulado. A partir da artéria renal principal surgia uma artéria polar aberrante que seguia um percurso de $55,59 \mathrm{~mm}$ desde sua origem até sua entrada no polo inferior do rim direito. Essa artéria aberrante tinha calibre de 2,65 $\mathrm{mm}$ e se iniciava $51,88 \mathrm{~mm}$ após a origem da artéria renal principal. Em um nível mais inferior, na altura da bifurcação da aorta, surgia outra artéria polar aberrante, de calibre 4,93 mm, com comprimento de 75,29 mm desde sua origem na aorta até sua entrada no hilo renal, seguindo um trajeto retilíneo até o polo inferior do rim direito (Figuras 1 e 2).

A segunda variação foi na quantidade das artérias hepáticas, bem como na origem das mesmas.

A artéria hepática comum originava-se no tronco celíaco e tinha um comprimento de 39,01 $\mathrm{mm}$ desde sua origem até sua entrada na porta do fígado. De sua origem no tronco celíaco até a origem de seu ramo gastroduodenal a distância era de 16,41 mm. A artéria mesentérica superior tinha origem no tronco celíaco e a distância entre sua origem e o surgimento da artéria hepática direita acessória era de $21,77 \mathrm{~mm}$. O comprimento da artéria hepática direita desde sua origem na artéria mesentérica superior até sua entrada na porta do fígado era de 71,99 mm (Figura 3).

\section{DISCUSSÃO}

Vários autores já determinaram variações anatômicas das arté-

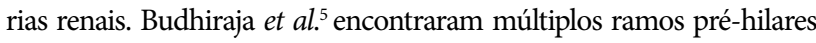


em $11,66 \%$ de sua amostra, duplicação da artéria renal em 8,33\% e artérias polares superiores em 6,66\% dos casos. Thakuria et al. ${ }^{9}$ encontraram, em um mesmo cadáver, quatro artérias variantes do lado direito e cinco artérias variantes do lado esquerdo, todas se originando da aorta. $\mathrm{Rao}^{7}$, em um estudo de caso, avaliou uma artéria polar aberrante direita que entrava na face anterior do rim, seguindo para o polo superior.

Budhiraja et al. ${ }^{8}$ encontraram uma variação semelhante à nossa com uma artéria renal aberrante originando-se na artéria

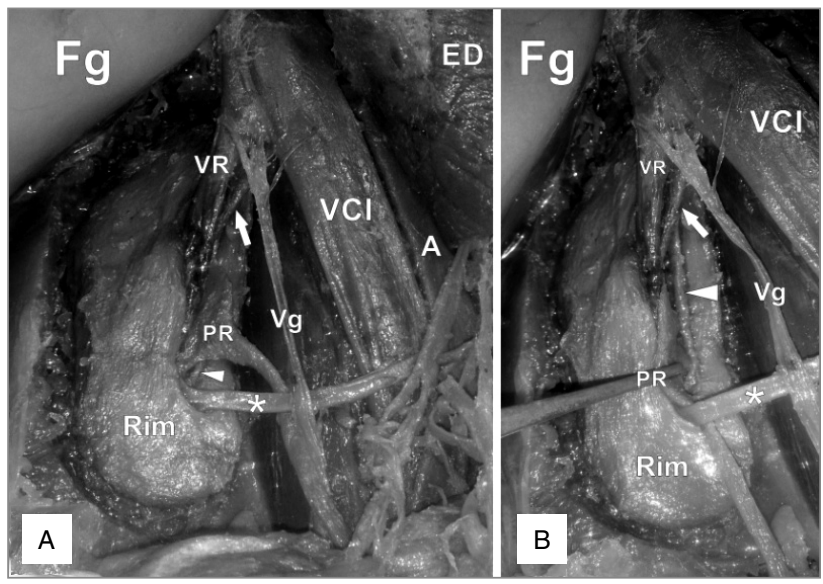

Figura 1: A e B: Fotografia do rim direito in situ. Fg: fígado; VR: veia renal direita; $\mathrm{VCl}$ : veia cava inferior; $\mathrm{A}$ : aorta; $\mathrm{PR}$ : pelve renal direita; Vg: veia gonadal direita; ED: estômago e duodeno rebatidos; seta: artéria renal direita; cabeça de seta: artéria renal polar aberrante; *artéria renal polar aberrante

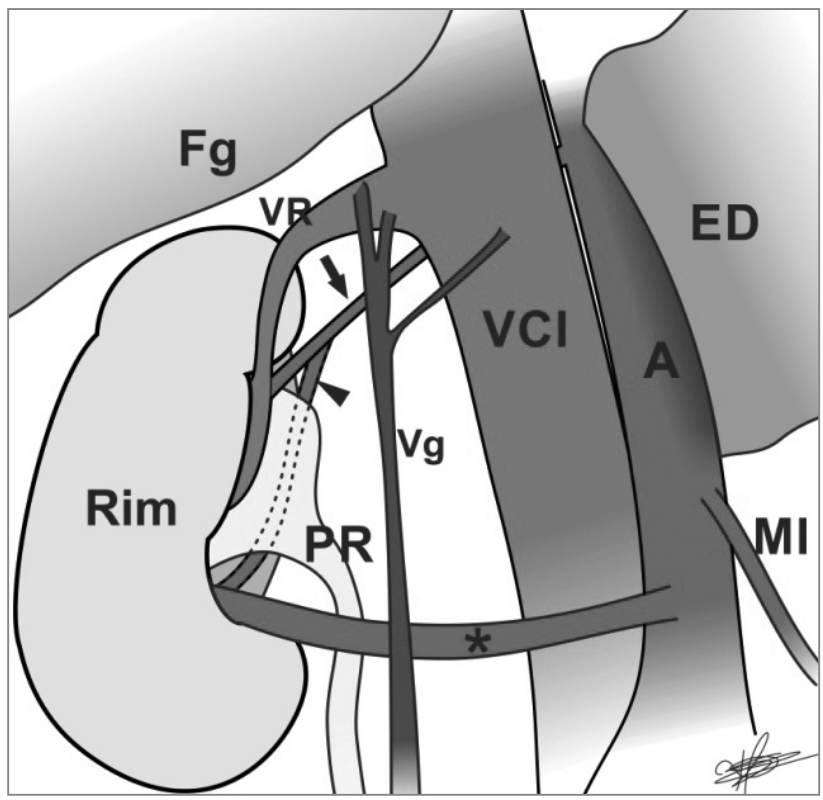

Figura 2: Ilustração esquemática das variações vasculares encontradas. Fg: fígado; VR: veia renal direita; VCl: veia cava inferior; A: aorta; PR: pelve renal direita; $\mathrm{Vg}$ : veia gonadal direita; ED: estômago e duodeno rebatidos; MI: artéria mesentérica inferior; seta: artéria renal direita; cabeça de seta: artéria renal polar aberrante; *artéria renal polar aberrante renal principal e entrando no polo inferior do rim e denominou-a de artéria polar inferior extra-hilar, bem como um ramo originando-se na aorta e entrando no polo inferior do rim em 4 de 42 espécimes estudados.

Patasi \& Boozary ${ }^{4}$ descreveram um caso semelhante ao nosso no que diz respeito a uma artéria renal acessória, originando-se abaixo da artéria mesentérica superior na aorta e seguindo diretamente para o polo inferior do rim direito.

Vários autores descreveram variações na origem e no trajeto da artéria hepática, inclusive criando classificações específicas ${ }^{10,14}$. Com o avanço dos métodos de imagem pudemos aumentar a quantidade de indivíduos estudados sem a necessidade da dissecação, fato que auxiliou exponencialmente os estudos quantitativos que definem um padrão das variações em determinadas populações. Em relação ao tronco celíaco, Ugurel et al. ${ }^{12}$ encontraram a formação tradicional do mesmo em 50de 100 pacientes $(50 \%)$. No que diz respeito à origem da artéria hepática comum a partir do tronco celíaco, o estudo de Hiatt et al. ${ }^{10}$ encontrou esse padrão em 75,7\% dos casos e Mitchels et al. ${ }^{14}$, em $27,5 \%$ dos casos.

No caso de uma artéria hepática direita acessória, os dados da literatura mostram que a incidência é bem menor: Michels et al. ${ }^{14}$ (9\%), Hiatt et al. ${ }^{10}(10,6 \%)$, Araujo Neto et al. ${ }^{13}$ (5,1\%), LópezAndújar et al. ${ }^{11}(0,6 \%)$.

Percebe-se que há uma grande quantidade e variedade de artérias renais acessórias e aberrantes, com diferentes medidas e trajetos. Existe uma vasta gama de ramos que suprem os polos superior e inferior dos rins, originando-se diretamente da aorta ou a partir da artéria renal principal, e isso é amplamente descrito na literatura, porém, não há consenso entre os diferentes autores sobre a nomenclatura utilizada na descrição de cada caso.
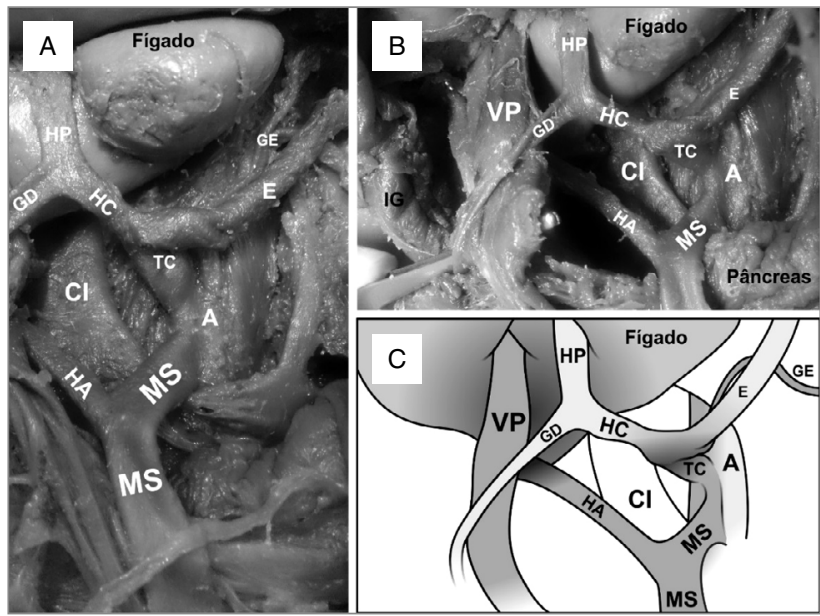

Figura 3: A e B: Fotografia dos vasos da região celíaca e sub-hepática; C: llustração esquemática da mesma região. HP: artéria hepática própria; HC: artéria hepática comum; GD: artéria gastroduodenal; $\mathrm{E}$ : artéria esplênica; GE: artéria gástrica esquerda; TC: tronco celíaco; $\mathrm{A}$ : artéria aorta; $\mathrm{MS}$ : artéria mesentérica superior; HA: artéria hepática acessória; IG: intestino grosso; $\mathrm{Cl}$ : veia cava inferior; VP: veia porta 
As variações da artéria hepática, bem como do tronco celíaco, têm sido extensamente descritas na literatura, porém, não há um padrão na quantidade de variações encontradas em conjunto entre o tronco celíaco e a artéria hepática comum, o que nos leva a uma necessidade de descrição a cada caso encontrado.
A qualificação e a quantificação das variações anatômicas constituem uma tarefa árdua e difícil em decorrência das inúmeras expressões diferentes que ocorrem no corpo humano, sendo que as porcentagens variam imensamente, podendo apresentar valores de menos de $1 \%$ ou chegar a valores maiores do que $50 \%$.

\section{REFERÊNCIAS}

1. Standring S. Gray's anatomy: the anatomical basis of clinical practice. $41^{\text {th }}$ ed. New York: Elsevier; 2015.

2. Tubbs RS, Shoja MM, Loukas M. Bergman's comprehensive Encyclopedia of human anatomic variation. Wiley-Blackwell; 2016. https://doi.org/10.1002/9781118430309

3. Ozkan U, Oğuzkurt L, Tercan F, Kizilkiliç O, Koç Z, Koca N. Renal artery origins and variations: angiographic evaluation of 855 consecutive patients. Diagn Interv Radiol. 2006;12(4):183-6.

4. Patasi B, Boozary A. A case report: accessory right renal artery. Int J Anatom Var. 2009;2:119-21.

5. Budhiraja V, Rastogi R, Asthana AK. Renal artery variations: embryological basis and surgical correlation. Rom J Morphol Embryol. 2010;51(3):533-6.

6. Budhiraja V, Rastogi R, Asthana AK. Variant origin of superior polar artery and unusual hilar branching pattern of renal artery with clinical correlation. Folia Morphol (Warsz). 2011;70(1):24-8.

7. Rao TR. Aberrant renal arteries and its clinical significance: a case report. Int J Anatom Var. 2011;4:37-9.

8. Budhiraja V, Rastogi R, Jain V, Bankwar V. Anatomical variations of renal artery and its clinical correlations: a cadaveric study from central India. J Morphol Sci. 2013;30(4):228-33.

9. Thakuria S, Roy RD, Baruah PD, Das TK. Multiple renal arteries: a case report. Int J Anatom Var. 2013;6:155-7.
10. Hiatt JR, Gabbay J, Busuttil RW. Surgical anatomy of the hepatic arteries in 1000 cases. Ann Surg. 1994;220(1):50-2.

11. López-Andújar R, Moya A, Montalvá E, Berenguer M, De Juan $M$, San Juan $F$, et al. Lessons learned from anatomic variants of the hepatic artery in 1,081 transplanted livers. Liver Transpl. 2007;13(10):1401-4.

http://dx.doi.org/10.1002/lt.21254

12. Ugurel MS, Battal B, Bozlar U, Nural MS, Tasar M, Ors F, et al. Anatomical variations of hepatic arterial system, coeliac trunk and renal arteries: an analysis with multidetector CT angiography. Br J Radiol. 2010;83(992):661-7.

http://dx.doi.org/10.1259/bjr/21236482

13. Araujo Neto AS, Franca HA, Mello Jr CF, Silva Neto EJ, Negromonte GRP, Duarte CMA, et al. Anatomical variations of the celiac trunk and hepatic arterial system: an analysis using multidetector computed tomography angiography. Radiol Bras. 2015;48(6):358-62

http://dx.doi.org/10.1590/0100-3984.2014.0100

14. Michels NA. Newer anatomy of the liver and its variant blood supply and collateral circulation. Am J Surg. 1966;112(3):337-47. http://dx.doi.org/10.1016/0002-9610(66)90201-7

15. Sebben GA, Rocha SL, Sebben MA, Parussolo Filho PR Gonçalves BH. Variações da artéria hepática: estudo anatômico em cadáveres. Rev Col Bras Cir. 2013;40(3):221-6. http://dx.doi.org/10.1590/S0100-69912013000300010 